\title{
The causal agent of anthracnose in papaya fruit and control by three different Malaysian stingless bee honeys, and the chemical profile
}

\begin{abstract}
Anthracnose is a fungal disease that leads to a serious quality problem on a wide range of fresh produce. The current study attempted to investigate the potential of antifungal activity in Malaysian stingless bee or kelulut honey $(\mathrm{KH})$ against anthracnose disease in papaya. $\mathrm{KH}$ was obtained from bee honey collectors from three different regions in Malaysia i.e Selangor, Kelantan and Sarawak. The screening for antifungal activity was performed via poison agar method using in vitro and in vivo assays against postharvest fungus Colletotrichum sp. The presence of the fungus was detected and confirmed by polymerase chain reaction (PCR) as well as a morphological technique. Results indicated $15 \%$ concentration of $\mathrm{KH}$ exhibited potent inhibitory effects and suppressed the mycelial growth of Colletotrichum sp. The scanning electron microscope (SEM) analysis revealed the rupture of the spores of fungus treated with $15 \%$ concentration of $\mathrm{KH}$ solution and degradation of hyphal growth. Further study was conducted on the $\mathrm{KH}$ solution for the detection of phenolic and flavonoid compounds by using high-performance liquid chromatography (HPLC) with a diode array detector. Results demonstrated that all of the samples contained gallic acid, coumaric acid, ferulic acid, and salicylic acid as phenolics compounds while naringenin and kaempferol as flavonoid compounds, which could contribute to the antifungal potential.
\end{abstract}

Keyword: Stingless bee honey; Kelulut; Postharvest disease; Colletotrichum sp.; Antifungal; Tropical fruits 